submitted for presentation at the 2005 AIAA Guidance, Navigation \& Control Conference

\title{
Adaptive Control for Microgravity Vibration Isolation System
}

\author{
Bong-Jun Yang;, Anthony J. Calise † James I. Craig † \\ School of Aerospace Engineering, Georgia Institute of Technology \\ Atlanta, GA 30332 \\ Mark S. Whorton $\S$ \\ Guidance, Navigation, and Mission Analysis, NASA Marshall Space Flight Center \\ Huntsville, $A L$
}

\begin{abstract}
Must active vibration isolation systems that try to a provide quiescent acceleration environment for space-science experiments have utilized linear design methods. In this paper, we address adaptive control augmentation of an existing classical controller that employs a high-gain acceleration feedback together with a low-gain position feedback to center the isolated platform. The control design feature includes parametric and dynamic uncertainties because the hardware of the isolation system is built as a payload-level isolator, and the acceleration sensor exhibits a significant bias. A neural network is incorporated to adaptively compensate for the system uncertainties, and a high-pass filter is introduced to mitigate the effect of the measurement bias. Simulations show that the adaptive control improves the performance of the existing acceleration controller and keeps the level of the isolated platform deviation to that of the existing control system.
\end{abstract}

\section{Introduction}

The low-acceleration environment on the International Space Station (ISS) enables to conduct new microgravity science experiments that are practically impossible on the surface of the Earth. A variety of vibro-acoustic disturbances on the ISS, however, are present and degrade the performance of many $\mu$ g experiments. As a matter of fact, the acceleration environment on the ISS is expected to exceed the requirements of many acceleration sensitive experiments ${ }^{1}$ as shown in Figure 1. By comparing the requirement with the expected ISS acceleration in Figure 1, an isolation performance specification can be derived. The isolation system must attenuate the ambient ISS acceleration by one order of magnitude at $0.1 \mathrm{~Hz}$, which

\footnotetext{
- Postdoctoral Fellow, jun.yang@ae.gatech.edu, AIAA member

$\dagger$ Professor, anthony.calise@ae.gatech.edu, AIAA Fellow

‡Professor, james.craig@ae.gatech.edu, Senior AIAA member

${ }^{5}$ Aerospace Engineer, mark.whorton@nasa.gov, Senior AIAA member
}

1 of 18 


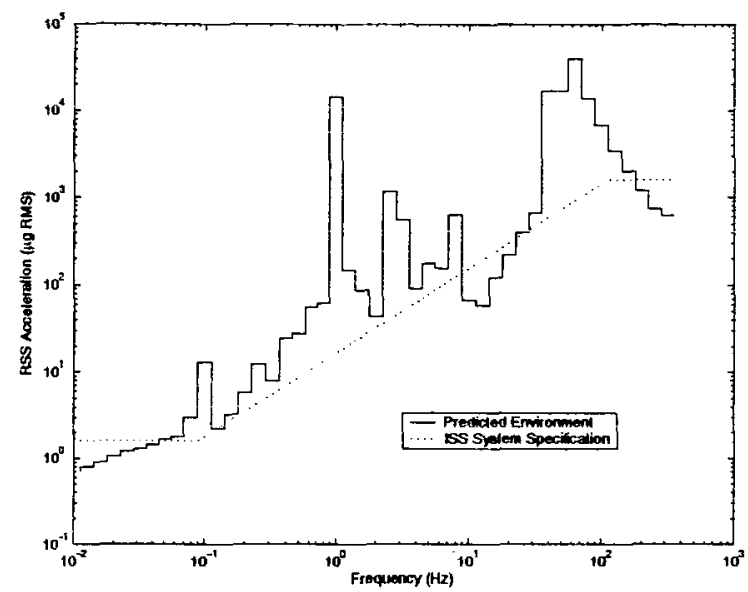

Figure 1. Predicted RMS Acceleration Environment of the International Space Station

for a second order system implies maximum break-frequency of $0.01 \mathrm{~Hz}$. That is, the isolated system must transmit the quasi-steady accelerations of the vehicle to the isolated assembly (below $0.01 \mathrm{~Hz}$ ) and attenuate disturbances above $0.01 \mathrm{~Hz}$. This performance specifications necessitate the implementation of active vibration isolation system at the payload/rack locations because passive isolation systems, in general, are not adequate to provide sufficient attenuation of vibration due to ubiquitous nature of disturbance sources.

An example of rack-level vibration isolation system is the Active Rack Isolation System (ARIS), ${ }^{2}$ the control architectures and Hight-test results of which can be found in [3,4]. In contrast to rack-level isolation systems, g-LIMIT (Glovebox Integrated Microgravity Isolation Technology) shown in Figure 2 is a micro-

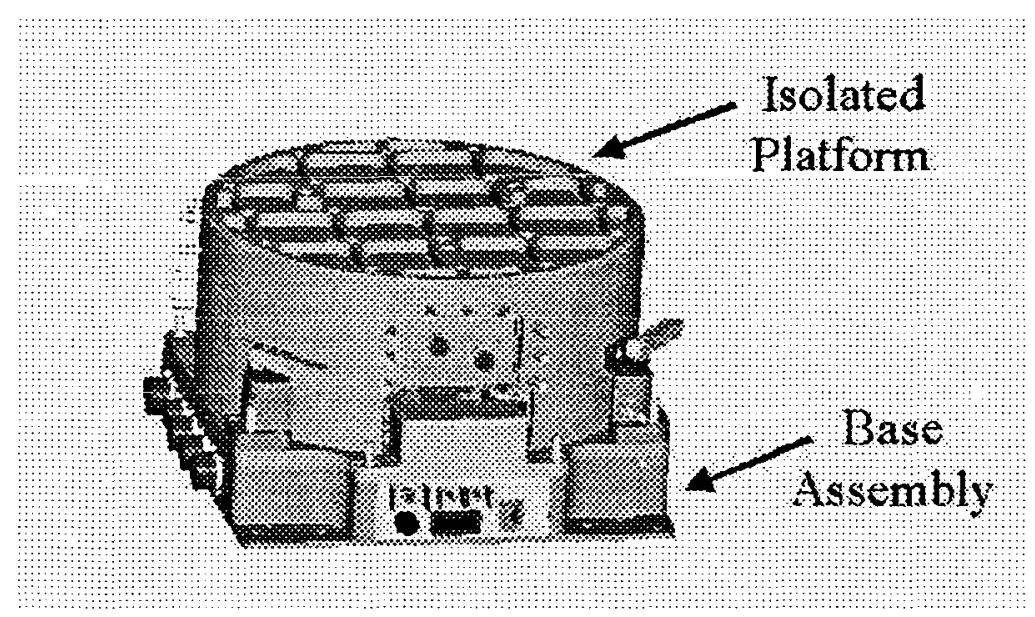

Figure 2. g-LIMIT System Assembly

gravity vibration isolation system that is designed to isolate experiments at the payload level. The g-LIMIT hardware consists of the inertially isolated assembly to which an experiment is mounted and the base assembly that is rigidly attached to the ISS rack support structure. In order to provide quiescent acceleration environment for an experiment, g-LMMT utilizes six independent control actuation channels that provide six 
independent magnetic forces to a platform upon which the experiment resides. g-LIMIT is designed around three integrated isolator modules (IM's), each of which is comprised of a dual-axis magnetic actuator, two axes of acceleration sensing, two axes of sensing the relative position of each IM from the ISS-mounted base assembly. ${ }^{5}$ Integrated into the base assembly and the isolated assembly is a snubber system, which provides mechanical rattle-space constraints with a maximum relative displacement of $1 \mathrm{~cm}$ between the isolated assembly and the base assembly. The only mechanical connection between the isolated platform and the base assembly is the set of umbilicals that pass resources between the ISS and the experiment.

The design of an isolation system for g-LIMTT is a challenging problem due to the stringent performance requirement and static and dynamic uncertainties that arise due to kinematic coupling between the platform and the mounted experiment and the damping and stiffness properties of umbilicals. The mass and inertia properties of the system change considerably as various experiments utilize the isolation system during its operation. Unlike the rack-level isolation system where the rack structure is much more massive than the individual experiment, mass and inertial variation in g-LMIT are generally the same size as the isolation system and thus more problematic. The umbilicals are primary load path for the ISS disturbances to the isolated system and primary source of uncertainties for control system design since the stiffness and damping properties of them cannot be accurately measured on the ground due to gravitational deflections and coupling. Moreover, the flight-test results in [3] reveals the possibility of hysteresis in their stiffness properties, which may become significant in low-amplitude acceleration environment and degrade the performance of the isolation system. ${ }^{3}$

Most vibration isolation systems have used linear control methods ${ }^{6-9}$ (an exception found in [10]). For the design of a control system for g-LIMIT, standard approaches have employed a two-loop (inner/outer) architecture. That is, a high-gain acceleration feedback is employed to cancel the accelerations in the innerloop, and a low-gain position feedback is added to the outer-loop to center the platform in the sway space and drive the platform to follow the quasi-steady motion of the vehicle. In [8], classical Proportional-IntegralDerivative (PID) controllers are designed for both the acceleration and position feedback. Fixed-order mixed $\mathrm{H}_{2} / \mu$ controller is considered for acceleration feedback in[9], a the nominal performance and robustness of which are compared to those of a standard $\mathrm{H}_{2}$ method.

In this paper, we consider adaptive control design that augments an PID control design in [8] to guarantee the performance under modelling uncertainties. The adaptive elements are designed following the method described in $[11,12]$. A neural network $(\mathrm{NN})$ is employed to approximately cancel the uncertainty. It is well established that a NN can approximate any continuous function to any desired accuracy, ${ }^{13}$ which has been a main reason for the use of a NN in many approaches. ${ }^{14-16}$ In an output feedback setting, the method that uses a memory unit of input/output delays to approximate an uncertainty has been proposed ${ }^{17,18}$ and shown to be effective in many output feedback applications. ${ }^{12,19-21}$ The method in [11] is selected for the design of adaptive control for g-LIMIT because an acceleration is the output that renders the system nonminimum

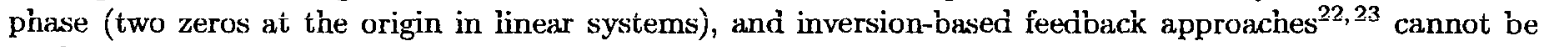
applied.

The paper is organized as follows: In Section I we present the probiem of a control design for g-LIMIT in a single-input single-output (SISO) setting as the problem of a control design for a single mass-spring-damper system on which an experiment, modelled as another mass-spring-damper system, is mounted. In Section II, the essential features of the existing control system in [8] are provided in a SISO setting. In Section III, the details of the augmenting adaptive controller design are given, with emphasis on how the approach in [11] is adapted to address the specific challenges that arise in this application. In Section IV, simulation results are described that support the validity of the overall approach. Conclusion and future research are given in Section V.

The presentation in the manuscript is given in the setting of a single-input single-out (SISO) mass-spring-damper system. On final submission, multi-input multi-ouput (MIMO) results will be presented together with simulation results for a 6 degree-of-freedom g-LIMIT simulation model. 


\section{Problem Formulation}

As a 1 degree-of-freedom SISO system, g-LMIT is considered as a single mass-spring-damper system. We consider a g-LIMIT on which a unknown flexible experiment is mounted as depicted in Figure 3 . The

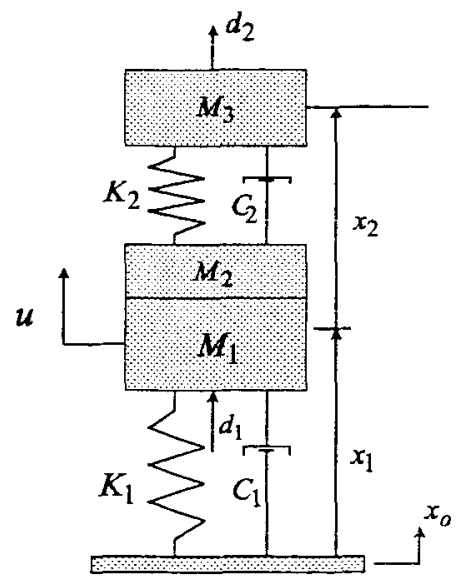

Figure 3. Mass Spring Damper with Unmodeled Dynamics

term $x_{0}$ represents the displacement of the base, $x_{1}$ is the relative displacement from the base, and $x_{2}$ stands for the relative displacement between $M_{2}$ and $M_{3}$. The equation of motion for the system in Figure 3 is given by

$$
\begin{aligned}
& M_{c}\left(\ddot{x}_{o}+\ddot{x}_{1}\right)+C_{1} \dot{x}_{1}+K_{1} x_{1}-C_{2} \dot{x}_{2}-K_{2} x_{2}=u+d_{1} \\
& M_{3}\left(\ddot{x}_{o}+\ddot{x}_{1}+\ddot{x}_{2}\right)+C_{2} \dot{x}_{2}+K_{2} x_{2}=d_{2},
\end{aligned}
$$

where $M_{c}=M_{1}+M_{2}$, and $d_{1}$ and $d_{2}$ stand for external disturbances applied to $M_{1}$ and $M_{3}$ respectively. Two measured outputs are the absolute acceleration of $M_{1}$ and the relative displacement $x_{1}$

$$
y_{1}=\ddot{x}_{o}+\ddot{x}_{1}, y_{2}=x_{1} \text {. }
$$

The parameters are: $M_{1}=20, C_{1}=0.5242, K_{1}=6.1574, M_{2}=5, M_{3}=15, C_{2}=1 \times 10^{-15}$, and $K_{2}=10$. Given the outputs in (2), with a state vector $x=\left[x_{1} \dot{x}_{1}, x_{2}, \dot{x}_{2}\right]^{T}$, it is straightforward to derive a state-space description for (1)

$$
\begin{aligned}
\dot{x} & =A x+B\left(u+d_{1}\right)+B_{f} \ddot{x}_{o}+B_{2} d_{2}, \quad x \in \mathbb{R}^{4 \times 1} \\
y_{1} & =C_{1} x+D u, \\
y_{2} & =C_{2} x .
\end{aligned}
$$

The plant model, which is used in the design of feedback control, consists of a single mass-spring-damper

$$
\hat{M}\left(\ddot{x}_{o}+\ddot{x}\right)+\hat{C} \dot{x}+\hat{K} x=u,
$$

where $\hat{M}=17.8488, \hat{C}=0.5242$, and $\hat{K}=6.1574$. With the outputs corresponding to those in (2), by letting $x_{m}=[x, \dot{x}]^{T}$, the plant model is expressed in the state-space form

$$
\begin{aligned}
\dot{x}_{m} & =A_{m} x_{m}+B_{m}\left(u+d_{1}\right)+B_{m_{j}} \ddot{x}_{o}, \quad x_{m} \in \mathbb{R}^{2 \times 1} \\
y_{m_{1}} & =C_{m_{1}} x_{m}+D_{m} u, \\
y_{m_{2}} & =C_{m_{2}} x_{m} .
\end{aligned}
$$


The frequency responses of the plant model are compared to those of the true system in (3) in Figure 4. The disturbance attenuation requirement that can be derived from Figure 1 is associated with the trañmissibility, $\frac{\ddot{x}}{\ddot{x}_{0}}=G_{a b}(s)$, from the base to the isolated assembly $M_{1}$ in Figure $4(\mathrm{c})$. The control system design generally involves $G_{a u}=\frac{y_{1}(s)}{u(s)}$ and $G_{x u}=\frac{y_{2}(s)}{u(s)}$ in Figures $4(\mathrm{a})$ and $4(\mathrm{~b})$. Note that the transfer functions from the input $u$ to the acceleration and the position are the same as those from the disturbance $\dot{d}_{1}$ to the acceleration and the position since $u$ and $d_{1}$ are applied in the same manner as seen in Figure 3.
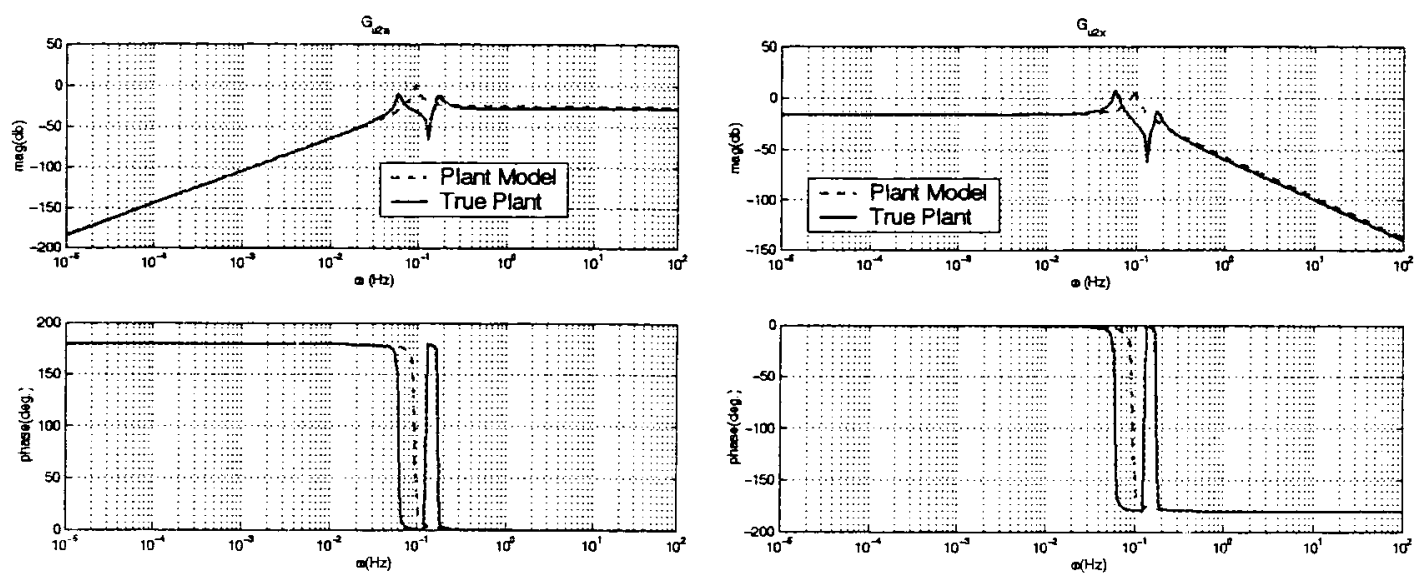

(a) Bode Flot for $G_{a u}\left(=\frac{y_{1}(s)}{u(s)}\right)$.

(b) Bode Plot for $G_{x u}\left(=\frac{y_{z}(s)}{u(s)}\right)$.
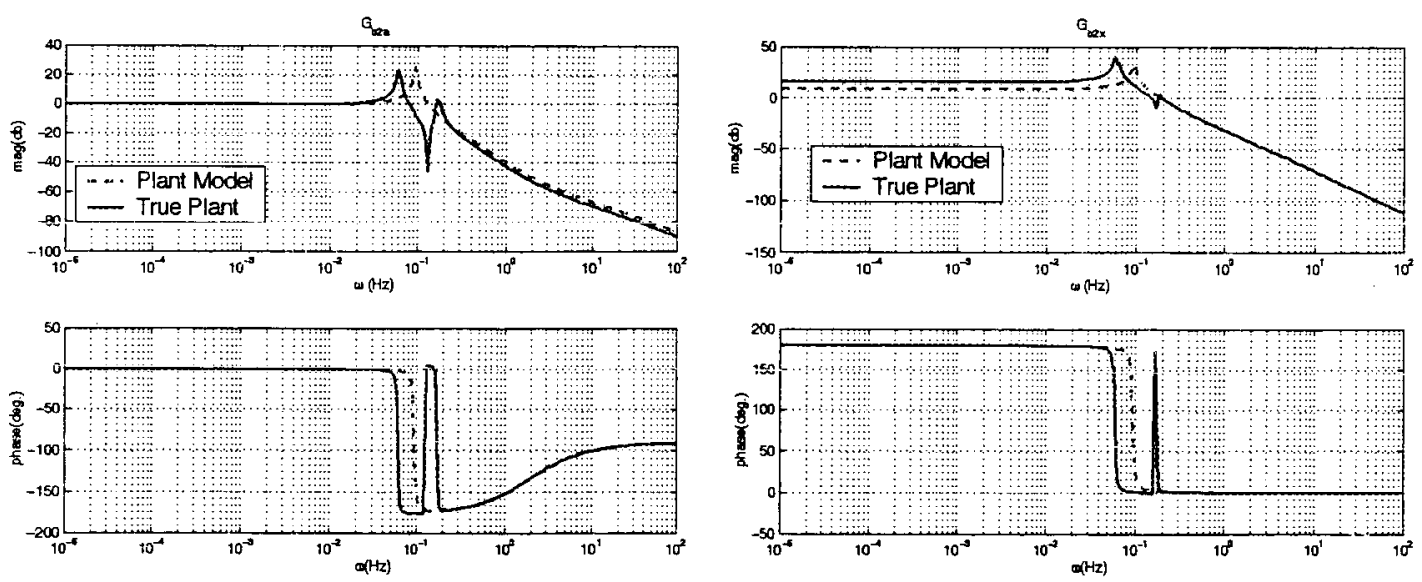

(c) Bode Plot for $G_{a b}\left(=\frac{y_{1}(s)}{\tilde{x}_{o}(s)}\right)$.

(d) Bode Plot for $G_{x b}\left(=\frac{y_{2}(s)}{x_{o}(s)}\right)$.

Figure 4. Frequency Response of Various Transfer Functions

The control objective of the vibration isolation system is to design a control law for $u$, using the plant model in (5), so as to cancel the acceleration of $M_{1}\left(y_{1}\right)$ by an acceleration feedback while centering $M_{1}$ (regulating $y_{2}$ ) by a position feedback when the control law is applied to the true system in (3). A standard 
approach for the objective is to incorporate two time-scale designs for acceleration and position feedback. That is, a high-gain, high-bandwidth acceleration feedback controller is designed for acceleration feedback to meet the acceleration requirement while a low-gain, low-bandwidth position feedback controller is utilized to maintain the position at the center in the sway space.

\section{Existing Control System}

The existing control system described in [8] is laid out in Figure 5, in which $x_{c}$ represents the commanded relative position (generally zero) and $n$ represents the accelerometer error (due to a sensor noise and a bias). The acceleration controller, an inner-loop controller $G_{a}(s)$ in Figure 5 , is designed as a PI controller

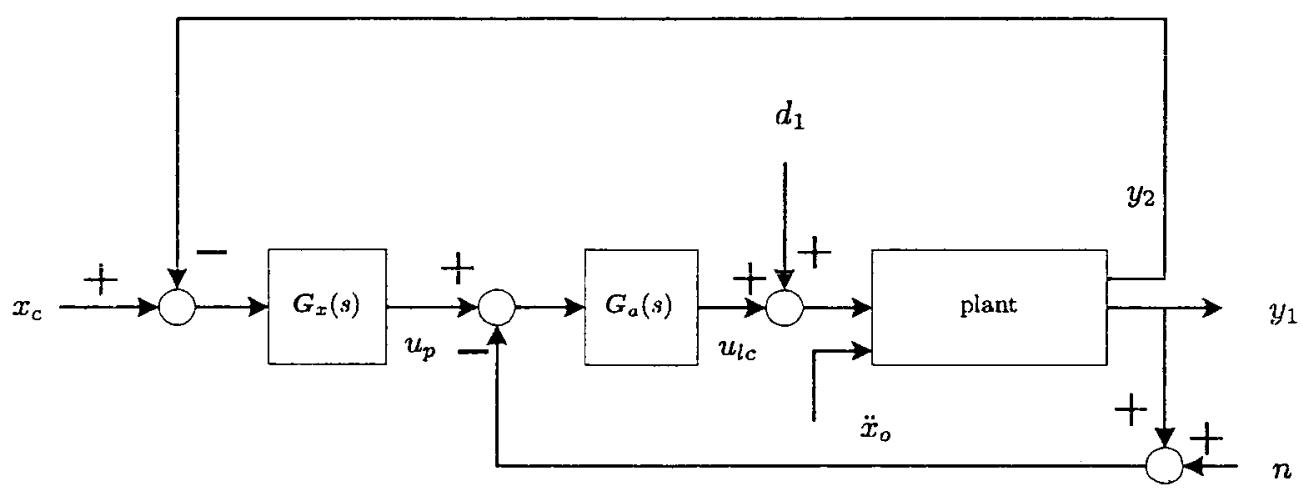

Figure 5. Existing Control System Architecture

$$
u_{\imath c}=C_{a}(s)\left[y_{c}-\left(y_{1}+n\right)\right]=\frac{K_{I_{i}}+K_{I_{p}} s}{s}\left[y_{c}-\left(y_{1}+n\right)\right]
$$

where $K_{I_{1}}=250$, and $K_{I_{p}}=0.4175$. In case the position feedback controller is not present, applying the controller in (6) to the plant model in (5) and the true plant in (3) leads to the frequency responses shown in Figure 6. Overall frequency responses reveal that the mounted experiment does not influence much on the isolation system. Figure 6(c) shows that the acceleration controller is designed to meet the required transmissibility. The frequency response from the sensor error to the relative position shown in Figure 6(d) reveals an undesirable effect of the sensor bias, which is an indispensable feature of the g-LIMIT accelerometer. The accelerometer bias in g-LIMIT is at the mili-g level, and Figure 6(d) shows that without compensation for this bias the platform deviates from the center of the sway space significantly. Together with a high-gain acceleration controller, this sensor bias $n$ renders the outer-loop position feedback necessary to move the isolated platform back to the center of the sway space.

The position controller, the outer-loop controller $G_{x}(s)$ in Figure 5, is designed as a PID controller in which the integral action is required to remove the effect of the accelerometer bias. Since direct implementation of derivative control amplifies the sensor noise and causes numerical problems, the PID controller is realized as

$$
u_{p}(s)=\left[K_{p}+K_{d} \frac{s}{\frac{1}{50} s+1}+\frac{K_{i}}{s}\right]\left(x_{c}-y_{2}\right),
$$

where $K_{i}=3 \times 10^{-6}, K_{d}=0.02063$, and $K_{p}=0.00054$. Figure 7 shows frequency responses of the overall closed system. On the whole, the effect of the unmodelled dynamics is negligible, implying the robustness of the inner/outer loop PID controllers in case of a SISO mass-spring-damper system. Figure 7(c) indicates that

\section{6 of 18}



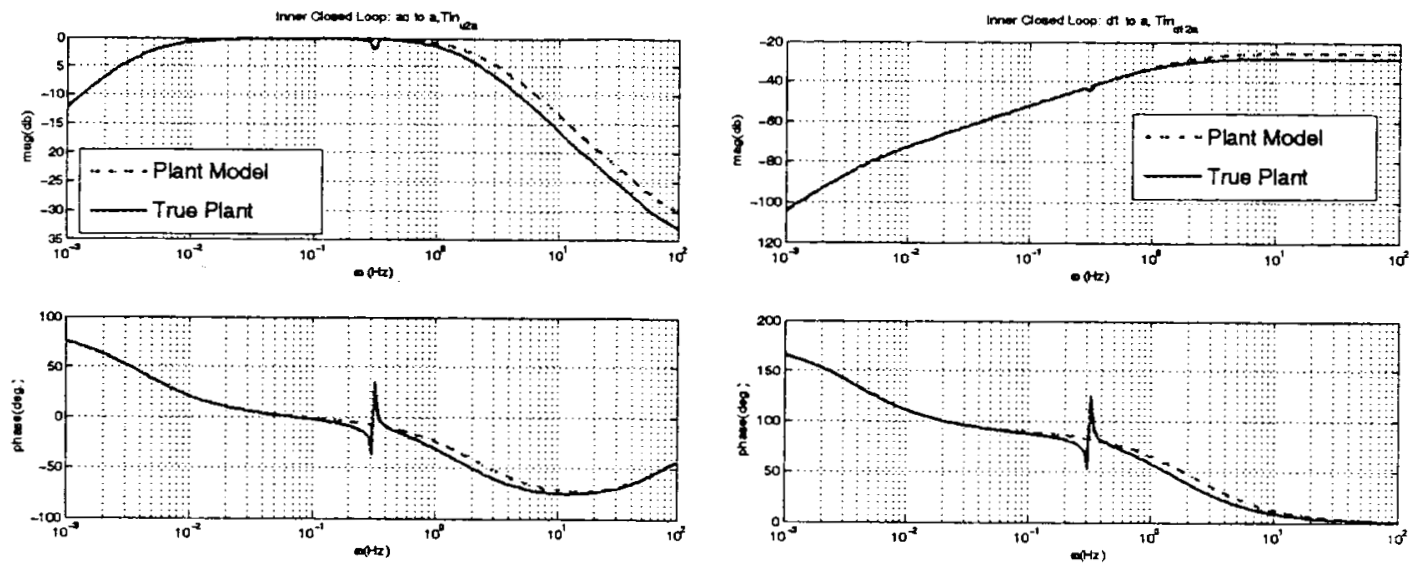

(a) Bode Plot for $\frac{y_{1}}{u_{p}}$.

(b) Bode Plot for $\frac{y_{1}}{d_{1}}$.
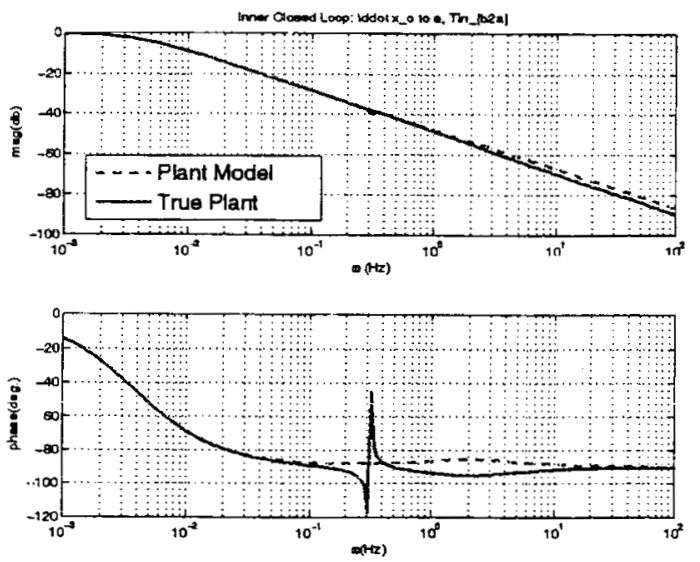

(c) Bode Plot for $\frac{y_{1}}{x_{0}}$.

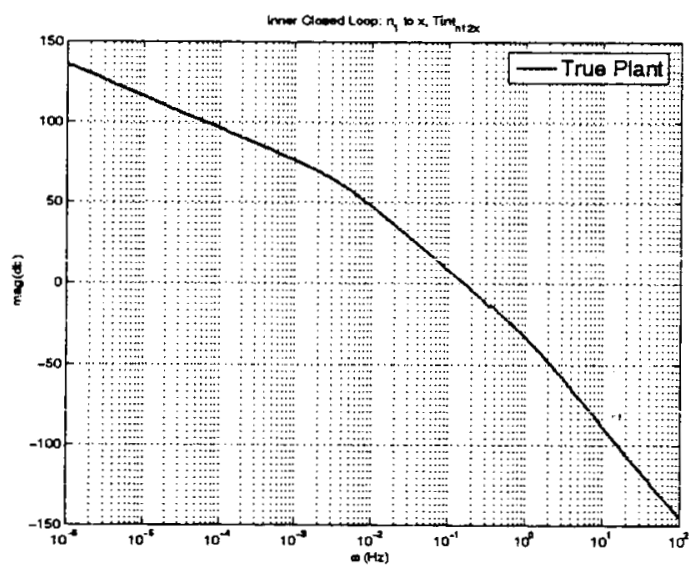

(d) Bode Plot for $\frac{y_{2}}{n}$.

Figure 6. Frequency responses for inner-loop transfer functions 

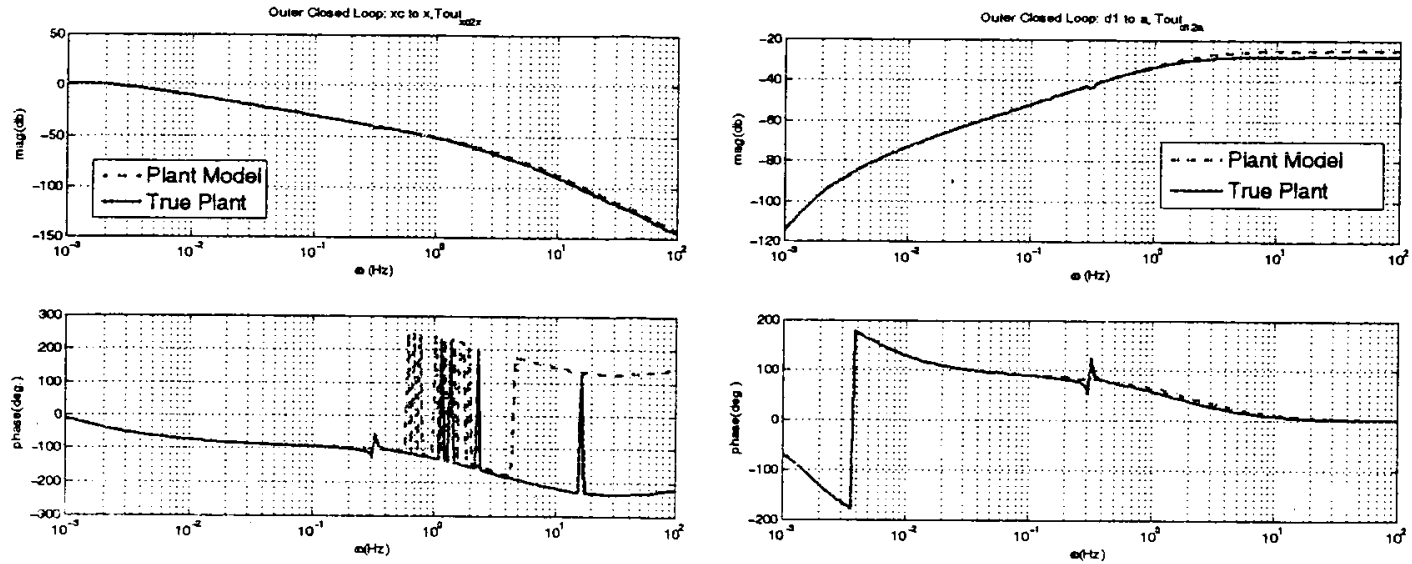

(a) Bode Plot for $\frac{y_{2}}{x_{c}}$

(b) Bode Plot for $\frac{y_{1}}{d_{1}}$.
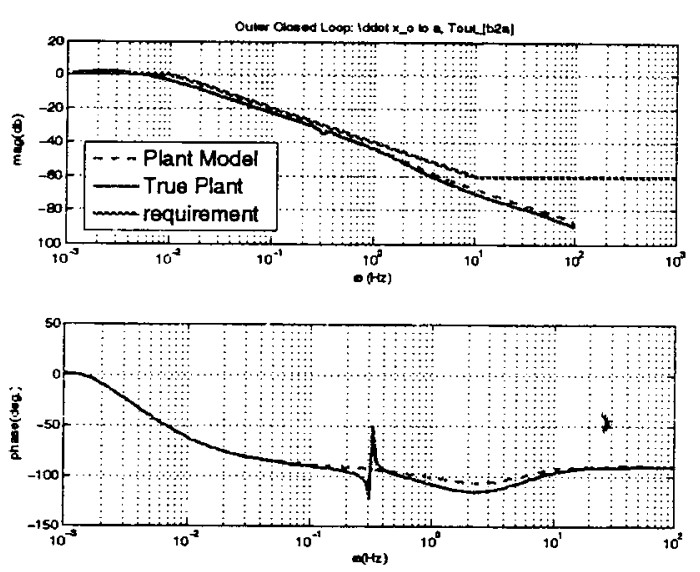

(c) Bode Plot for $\frac{y_{1}}{\ddot{x}_{0}}$.

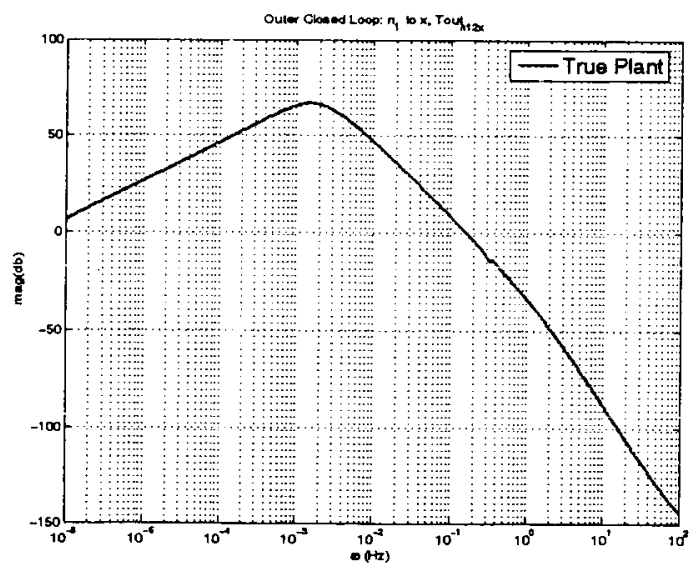

(d) Bode Plot for $\frac{y_{2}}{n}$.

Figure 7. Frequency responses for overall (inner-loop+outer-loop) transfer functions 
the existing control system meets the performance requirement for vibration isolation. That is, if the base excitations are the only source of external disturbances, which are transmitted by the spring, the performance of the isolation system is acceptable. Figure $7(\mathrm{~d})$ indicates that the outer-loop position controller can remove the effect of the accelerometer bias on the position in steady-state (The positive slope at the low-frequency region means zero gain at $0 \mathrm{~Hz}$ ).

\section{Adaptive Control Augmentation}

The purpose of the existing control system is to attenuate the accelerations and the only role of the lowgain outer-loop position controller is to remove the effect of the accelerometer bias and center the platform. Thus we augment the inner-loop acceleration controller using the method in [11] as depicted in Figure 8.

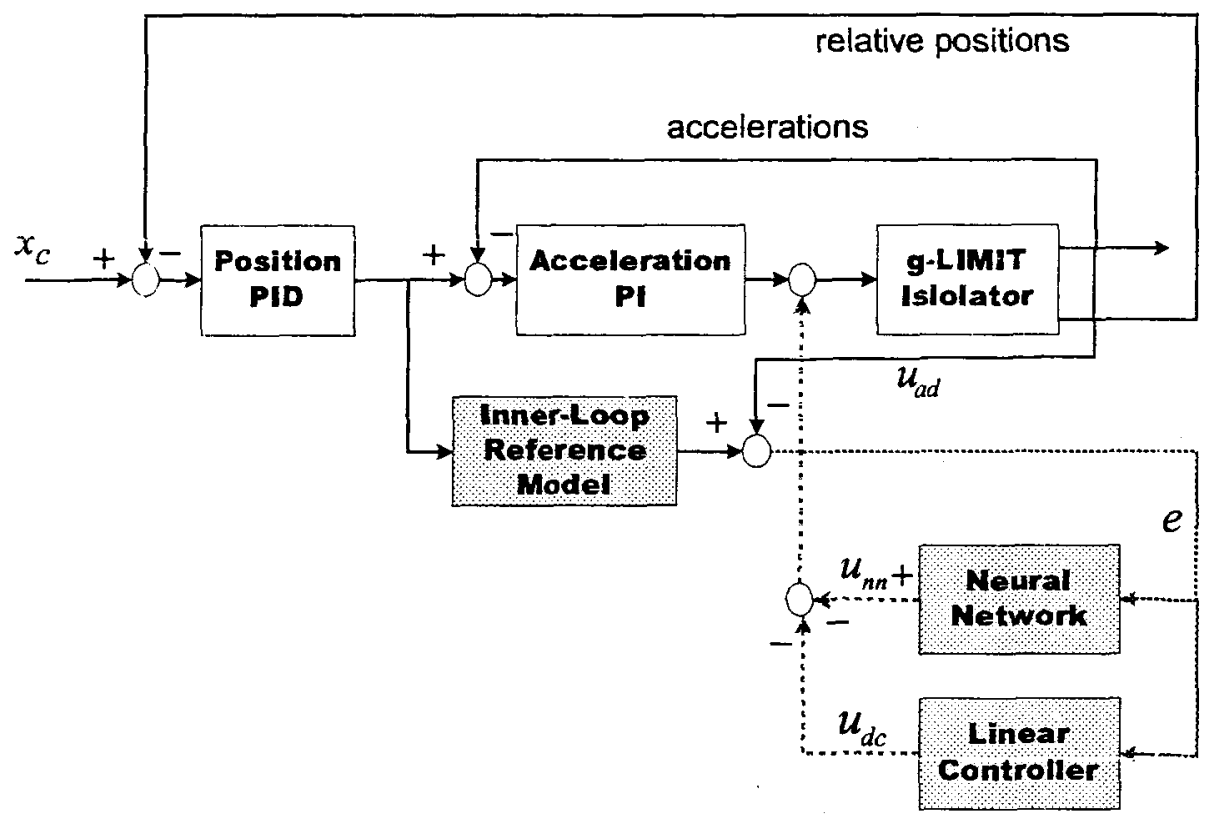

Figure 8. Closed Inner-Loop Reference Model based Augmentation Architecture

The immediate issue in this attempt is that the acceleration $y_{1}$ has the relative degree 0 and the method in [11], which assumes the relative degree greater than 1, cannot be applied. To circumvent this problem, we introduce an integrator before control input to achieve a relative degree 1 system ("dynamic extension" 24) when augmenting an adaptive control. With the dynamic extension, $\dot{\eta}=v, u=\eta$, the system in (3) is rewritten as

$$
\begin{aligned}
& \dot{x}_{a}=A_{a} x_{a}+B_{a} v+B_{a_{f}} \ddot{x}_{o}+B_{a_{d}} d \\
& y_{1}=C_{a_{1}} x_{a} \\
& y_{2}=C_{a_{2}} x_{a}
\end{aligned}
$$


where

$$
\begin{aligned}
& x_{a}=\left[\begin{array}{l}
x \\
\eta
\end{array}\right], d=\left[\begin{array}{l}
d_{1} \\
d_{2}
\end{array}\right], A_{a}=\left[\begin{array}{ll}
A & B \\
0 & 0
\end{array}\right], B_{a}=\left[\begin{array}{l}
0 \\
1
\end{array}\right], B_{a_{f}}=\left[\begin{array}{c}
B_{f} \\
0
\end{array}\right], B_{a_{d}}=\left[\begin{array}{cc}
B & B_{2} \\
0 & 0
\end{array}\right] \\
& C_{a_{1}}=\left[\begin{array}{ll}
C_{1} & D
\end{array}\right], C_{a_{2}}=\left[\begin{array}{ll}
C_{2} & 0
\end{array}\right] .
\end{aligned}
$$

In the same manner, with $\ddot{x}_{o}=d_{1}=0$, the extended plant model corresponding to ( 8 ) is described by

$$
\begin{aligned}
& \dot{x}_{a_{m}}=A_{a_{m}} x_{a_{m}}+B_{a_{m}} v, \\
& y_{m_{1}}=C_{a_{m_{1}}} x_{a_{m}}, \\
& y_{m_{2}}=C_{a_{m_{2}}} x_{a_{m}},
\end{aligned}
$$

where

$$
\begin{aligned}
& x_{a_{m}}=\left[\begin{array}{c}
x_{m} \\
\eta
\end{array}\right], A_{a_{m}}=\left[\begin{array}{cc}
A_{m} & B_{m} \\
0 & 0
\end{array}\right], B_{a_{m}}=\left[\begin{array}{l}
0 \\
1
\end{array}\right], \\
& C_{a_{m_{1}}}=\left[\begin{array}{ll}
C_{m_{1}} & D_{m}
\end{array}\right], C_{a_{m_{2}}}=\left[\begin{array}{ll}
C_{m_{2}} & 0
\end{array}\right] .
\end{aligned}
$$

\section{A. Inner-Loop Reference Model}

The inner-loop reference model in Figure 8 consists of the plant model in (5) regulated by tbe PI controller in (6) with $\ddot{x}_{o}=d_{1}=0$. The inner-loop reference model can also be viewed as the closed-loop system of the extended plant model in (10) and the PD controller as shown in Figure 9. To apply the method in [11], the

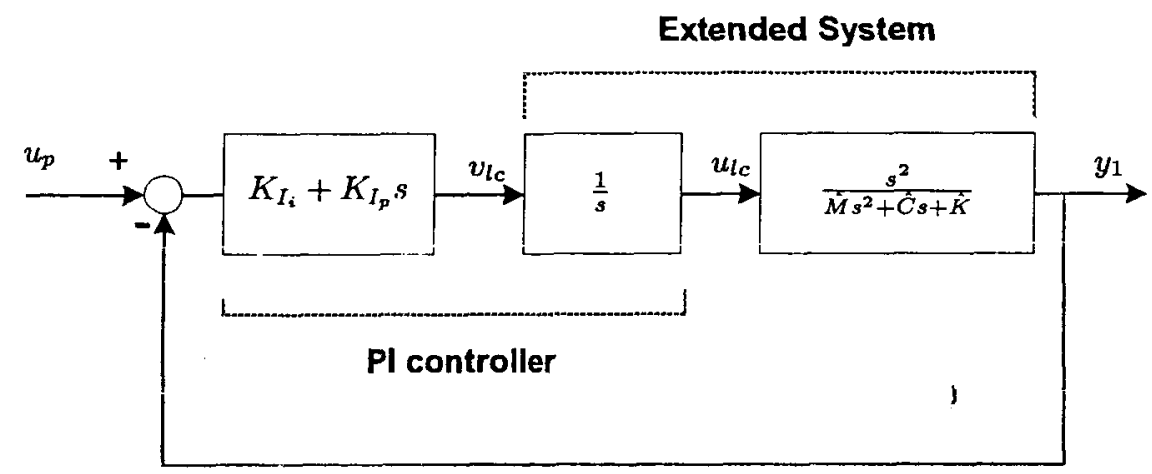

Figure 9. Inner-Loop Reference Model

system in (10) is transformed into a normal form ${ }^{24}$

$$
\begin{aligned}
\dot{y}_{m_{1}} & =a_{1} y_{m_{1}}+h_{o} z_{m}+D_{m} v \\
\dot{z}_{m} & =G_{o} y_{m_{1}}+F_{o} z_{m},
\end{aligned}
$$

where $z_{m}=\left[\begin{array}{ll}x_{1} & x_{2}\end{array}\right]^{T}$. The signal $v_{l c}\left(=\dot{u}_{c l}\right)$ is obtained by solving the following algebraic loop

$$
\begin{aligned}
v_{l c}=K_{I_{i}}\left(u_{p}-y_{m_{1}}\right)+K_{I_{p}}\left(\dot{u}_{p}-\dot{y}_{m_{1}}\right) & \\
= & K_{I_{i}}\left(u_{p}-C_{a_{m_{1}}} x_{a_{m}}\right)+K_{I_{p}}\left(\dot{u}_{p}-C_{a_{m_{1}}} A_{a_{m}} x_{a_{m}}-C_{a_{m_{1}}} B_{a_{m}} v_{l c}\right) . \\
& \frac{10 \text { of } 18}{\text { American Institute of Aeronautics and Astronautics }}
\end{aligned}
$$


This leads to

$$
v_{l c}=-\frac{1}{1+K_{I_{p}} D_{m}}\left[K_{I_{i}}+K_{I_{p}} a_{1}\right] y_{m_{1}}-\frac{K_{I_{p}}}{1+K_{I_{p}} D_{m}} h_{0} z_{m}+\frac{1}{1+K_{I_{p}} D_{m}}\left[K_{I_{i}} u_{p}+K_{I_{p}} \dot{u}_{p}\right] .
$$

Applying the control signal $v_{l c}$ in (14) to the normal-form extended plant model in (12) leads to the following inner-loop reference model

$$
\dot{\zeta}_{m}=\tilde{A} \zeta_{m}+\bar{B}_{p}\left[\begin{array}{l}
u_{p} \\
\dot{u}_{p}
\end{array}\right]
$$

where $\zeta_{m}=\left[\begin{array}{ll}y_{m_{1}} & z_{m}\end{array}\right]^{T}$, and

$$
\bar{A}=\left[\begin{array}{cc}
a_{1}-\frac{D_{m}\left(K_{I_{i}}+K_{I_{p}} a_{1}\right)}{1+K_{I_{p}} D_{m}} & 1-\frac{K_{I_{p}}}{1+K_{I_{p}} D_{m}} \\
G_{o} & F_{o}
\end{array}\right], \bar{B}_{p}=\left[\begin{array}{cc}
\frac{K_{I_{i}}}{1+K_{I_{p}} D_{m}} & \frac{K_{I_{p}}}{1+K_{I_{p}} D_{m}} \\
0 & 0
\end{array}\right]
$$

\section{B. Error Dynamics}

Compared to the extended plant model in (12), the extend system in (8) can be written in normal form

$$
\begin{aligned}
& \dot{y}_{1}=a_{1} y_{1}+h_{o} z_{1}+D_{m}\left(v+\Delta_{1}\right) \\
& \dot{z}_{1}=G_{o} y_{1}+F_{o} z_{1}+\Delta_{2}\left(y_{1}, z_{1}, z_{2}\right) \\
& \dot{z}_{2}=F_{2} z_{2}+F_{1} z_{1}+G_{1} y_{1} .
\end{aligned}
$$

where $z_{1}$ represents corresponding states to $z_{m}$ in (12) and $z_{2}$ results from the stable unmodelled dynamics. The unmatched uncertainty $\Delta_{2}=\dot{z}_{1}-G_{o} y_{1}-F_{o} z_{1}$, in general, satisfy the linear growth assumption in linear systems that is required for the stability proof. ${ }^{11}$ The matched uncertainty that will be compensated for by the adaptive elements is defined by

$$
\Delta_{1}\left(y_{1}, z_{1}, z_{2}, v\right)=\frac{1}{D_{m}}\left[C_{a_{1}} A_{a} x_{a}+C_{a_{1}} B_{a} v+C_{a_{1}} B_{a_{f}} \ddot{x}_{o}-\left[a_{1} y_{1}+h_{o} z+D_{m} v\right]\right] .
$$

Let

$$
v=v_{i c}+v_{u a} \text {. }
$$

The signal $v_{l c}\left(=\dot{u}_{l_{c}}\right.$, the PD control signal for the extended system in (17)) can be derived in the same manner as the inner-loop reference model is derived

$$
\begin{aligned}
v_{l c}= & -\frac{1}{1+K_{I_{p}} D_{m}}\left[K_{I_{i}}+K_{I_{p}} a_{1}\right] y_{1}-\frac{K_{I_{p}}}{1+K_{I_{p}} D_{m}} h_{o} z_{1}+\frac{1}{1+K_{I_{p}} D_{m}}\left[K_{I_{\mathbf{i}}} u_{p}+K_{I_{p}} \dot{u}_{p}\right]+ \\
& \frac{K_{I_{p}} D_{m}}{1+K_{I_{p}} D_{m}}\left[-v_{a d}-\Delta_{1}\left(y_{1}, z_{1}, z_{2}, v\right)\right] .
\end{aligned}
$$

Applying $v_{l c}$ in (20) to the system in (17) results in the following closed loop system

$$
\begin{aligned}
& \dot{\zeta}=\bar{A} \zeta+\bar{B}_{p}\left[\begin{array}{c}
u_{p} \\
\dot{u}_{p}
\end{array}\right]+\bar{B}\left(v_{a d}+\Delta_{1}\right)+\Delta_{2} \\
& \dot{z}_{2}=F_{2} z_{2}+F_{1} z_{1}+G_{1} y_{1}
\end{aligned}
$$

where

$$
\bar{B}=\left[\begin{array}{c}
1-\frac{K_{I_{p}}}{1+K_{I_{p}} D_{m}} \\
0
\end{array}\right] D_{m}, \Delta_{2}=\left[\begin{array}{c}
0 \\
\Delta_{2}
\end{array}\right]
$$


By defining the error vector as

$$
E=\zeta_{m}-\zeta
$$

we can derive the following error dynamics

$$
\begin{aligned}
& \dot{E}=\bar{A} E-\bar{B}\left(v_{a d}+\Delta_{1}\right)-\Delta_{2} \\
& \dot{z}_{2}=F_{2} z_{2}+F_{1} z_{1}+G_{1} y_{1} \\
& e_{1}=y_{m_{1}}-y_{1}=\bar{C} E
\end{aligned}
$$

where $\bar{C}=\left[\begin{array}{ll}1 & 0\end{array}\right]$.

\section{Linear Controller}

The eigenvalues of the matrix $\bar{A}$ are located at $-13.6905,-0.0246$, and 0 . The pole located at the origin results from the integrator in the PI controller. Since the approach in [11] requires $\bar{A}$ to be Hurwitz, we add an additional controller to stabilize the error dynamics in (24). Let

$$
v_{a d}=-v_{d c}-v_{n n}
$$

where the additional linear controller is designed as an linear quadratic Gaussian compensator

$$
\begin{gathered}
\dot{x}_{d c}=A_{d c} \boldsymbol{x}_{d c}+b_{d c} e_{1} \\
v_{d c}=C_{d c} \boldsymbol{x}_{d c}+d_{d c} e_{1} .
\end{gathered}
$$

Combining the compensator in (26) with the error dynamics in (24) leads to the closed-loop error dynamics

$$
\dot{E}_{a}=\bar{A}_{a} E_{a}+\bar{B}_{a}\left(v_{n n}-\Delta_{1}\right)-\left[\begin{array}{c}
\Delta_{2} \\
0
\end{array}\right],
$$

where

$$
\bar{A}_{a}=\left[\begin{array}{cc}
\bar{A}+\bar{B} d_{d c} \bar{C} & \bar{B} C_{d c} \\
d_{d c} \bar{C} & A_{d c}
\end{array}\right], \bar{B}_{a}=\left[\begin{array}{c}
\bar{B} \\
0
\end{array}\right] .
$$

The eigenvalues of $\bar{A}_{a}$ are $-13.6905,-13.6897,-2.35,-0.3332,-0.0069$, and -0.0006 . Since $\bar{A}_{a}$ is Hurwitz, for any $Q_{a}>0$, there exists a $P_{a}>0$ such that

$$
\bar{A}_{a} P_{a}+P_{a} \bar{A}_{a}+Q_{\tilde{a}}=0 .
$$

\section{High-Pass Filter and Adaptive Law}

A single hidden-layer $\mathrm{NN}$ is used to approximate $\Delta_{1}$ in (18), which is a function of states and control. The result in [18] establishes a universal approximation for an unknown function of states in an bounded observable process using a memory unit of sampled input/output pair. For arbitrary $\epsilon^{*}>0$, there exist bounded constant weights $\boldsymbol{M}, N$ such that:

$$
\Delta_{1}=M^{T} \sigma\left(N^{T} \eta\right)+\varepsilon(\eta),\|\varepsilon(\eta)\| \leq \epsilon^{*},
$$

where $\varepsilon(\eta)$ is the NN reconstruction error and $\eta$ is the network input vector

$$
\begin{aligned}
\eta(t) & =\left[\begin{array}{lll}
1 & \overline{\mathbf{u}}_{d}^{T}(t) & \overline{\boldsymbol{y}}_{d}^{T}(t)
\end{array}\right]^{T} \\
\overline{\boldsymbol{u}}_{d}^{T}(t) & =\left[\begin{array}{lll}
u(t) & u(t-d) & \cdots u\left(t-\left(n_{1}-r-1\right) d\right)
\end{array}\right]^{T} \\
\overline{\boldsymbol{y}}_{d}^{T}(t) & =\left[\begin{array}{lll}
y(t) & y(t-d) \cdots y\left(t-\left(n_{1}-1\right) d\right)
\end{array}\right]^{T}
\end{aligned}
$$


in which $n_{1}$ is the length of the window and generally required to be greater or equal to the system dimension, $d>0$ is a time-delay, $r$ is the relative degree of the output, $\sigma$ is a vector of squashing functions $\sigma(\cdot)$, its $i^{\text {th }}$ element being defined as $\left[\sigma\left(N^{T} \eta\right)\right]_{i}=\sigma\left[\left(N^{T} \eta\right)_{i}\right]$. In simulation, 4 delayed valies of $y_{1}$ in $(2)$ and 3 delayed values of the input $u$, with time delay $d=0.02$ sec., are combined to construct the NN input signal. The squashing functions are chosen as sigmoidal functions

$$
\left[\sigma\left(N^{T} \eta\right)\right]_{i}=\frac{1}{1+e^{-\alpha\left(N^{T} \eta\right)_{i}}}, i=1, \ldots, 6
$$

where $a=1$ represents the activation potential.

The adaptive signal $v_{n n}$ is designed as

$$
v_{n n}=\hat{M}^{T} \sigma\left(\hat{N}^{T} \eta\right)
$$

where $\hat{\boldsymbol{M}}$ and $\hat{N}$ are estimates of $M$ and $N$ to be adapted on-line. The adaptive law for the NN weights are the same as in [21] in which the output has a relative degree 1.

$$
\begin{aligned}
\dot{\hat{M}} & =-\Gamma_{M}\left[\left(\hat{\sigma}-\hat{\sigma}^{\prime} \hat{N}^{T} \eta\right) e P_{11} D_{m}+k \hat{M}\right] \\
\dot{\hat{N}} & =-\Gamma_{N}\left[e P_{11} D_{m} \eta \hat{M}^{T} \hat{\sigma}^{\prime}+k \hat{N}\right],
\end{aligned}
$$

in which $\Gamma_{M}, \Gamma_{N}>0$ are positive definite adaptation gain matrices, $k>0$ is a $\sigma$-modification constant, $\hat{\sigma} \triangleq \sigma(\hat{N} \eta), \hat{\sigma}^{\prime}$ is the Jacobian computed at the estimates, and $P_{11}$ is obtained from the decomposition of $P_{a}$ in (29) as follows:

$$
P_{a}=\left[\begin{array}{ll}
P_{11} & P_{12} \\
P_{12}^{T} & P_{22}
\end{array}\right], \quad P_{11} \in \mathbb{R}, P_{22} \in \mathbb{R}^{6 \times 6} .
$$

The adaptive law in (34) becomes, however, problematic when the measured output $y_{1}$ has a significant bias as is the case for the g-LIMIT accelerometer. The adaptive controller forces the biased measurement to track the output of the inner-loop reference model and leads to a large deviation of the isolation system from the center of the rattle-space. This is due to integral action in acceleration feedback that integrates the sensor bias. To remedy this situation, we introduce the following high-pass filter

$$
e_{f}=\frac{s}{s+\omega_{h}} e_{1}, \quad \omega_{h}=0.05 H z
$$

for the teaching signal $e_{1}$ and use $e_{f}$ instead for a teaching signal so that the NN adaptation takes place only to high-frequency error signals. Figure 10 represents the frequency response of the introduced high-pass filter. The resulting NN update laws are modified as

$$
\begin{aligned}
\dot{\hat{M}} & =-\Gamma_{M}\left[\left(\hat{\sigma}-\hat{\sigma}^{\prime} \hat{N}^{T} \eta\right) e_{f} P_{11} D_{m}+k \hat{M}\right] \\
\dot{\hat{N}} & =-\Gamma_{N}\left[e_{f} P_{11} D_{m} \eta \hat{M}^{T} \hat{\sigma}^{\prime}+k \hat{N}\right] .
\end{aligned}
$$

The following parameters are used for the adaptive law in (37)

$$
\Gamma_{M}=5 I, \Gamma_{N}=5 I, k=0.5
$$

where $I$ represents the identity matrix with a compatible dimension.

\section{Simulation Results}

In simulation, the reference command $x_{c}$ is set to zero. As Figure 7 suggests, the unmodelled dynamics have small influence on overall stability and performance. The accelerometer bias for $y_{1}$ is set as $0.001 \mathrm{~m} / \mathrm{sec}$ 

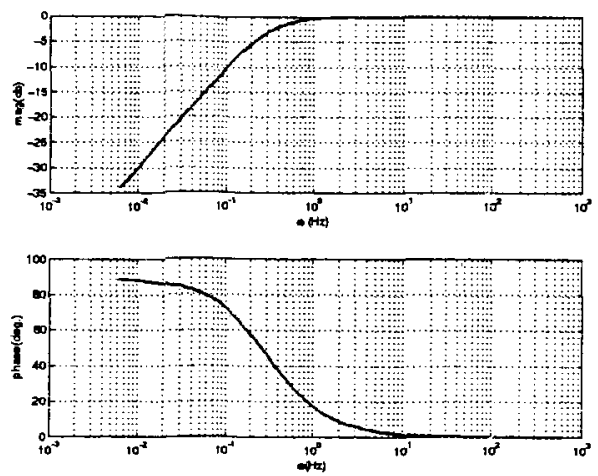

Figure 10. Frequency Response of the High-Pass Filter

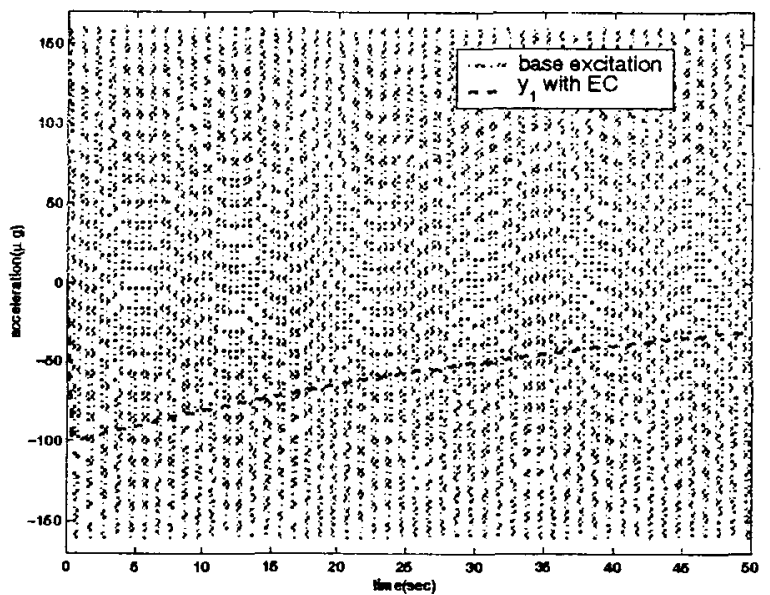

Figure 11. Time Responses of the base excitation $\ddot{x}_{o}$ and the acceleration $y_{1}$

$(=102 \mu \mathrm{g})$. Figure 11 shows the acceleration response $y_{1}$ when the base excitation $\ddot{x}_{o}$ is $160 \mu g \sin (2 \pi t)$. Figure 11 reveals that the base acceleration transmitted by the base is significantly attenuated by the existing isolation system as implied by the transmissibility in Figure 7 (c). The benefit of the adaptive control in enhancing the isolation performance can be seen in Figure 12. When the NN is implemented with its weights being updated as in (34) ("NN"), the vibration is further suppressed significantly, however, the isolated platform exhibit a drastic deviation in its position. When the high-pass filter in (36) is introduced for the teaching signal ("NN with HP"), Figure 12(b) shows that the relative position is regulated at the same level as the existing control system. Figure 13 shows the effect of the high-pass filter in (36) in tracking error. Without the high-pass filter, the acceleration integral controller keeps integrating the bias even when the true acceleration perfectly matches that of the inner-loop reference model and hinders the true acceleration from tracking the output of the inner-loop reference model as shown in Figure 13(a). In contrast, the highpass filter keeps the NN from seeing the sensor bias as the tracking error by removing the bias effect in the teaching signal and forces the truce acceleration to track the output of the inner-loop reference model as shown in Figure 13(b).

The assessment of the isolation performance throughout the overall frequencies requires the development 


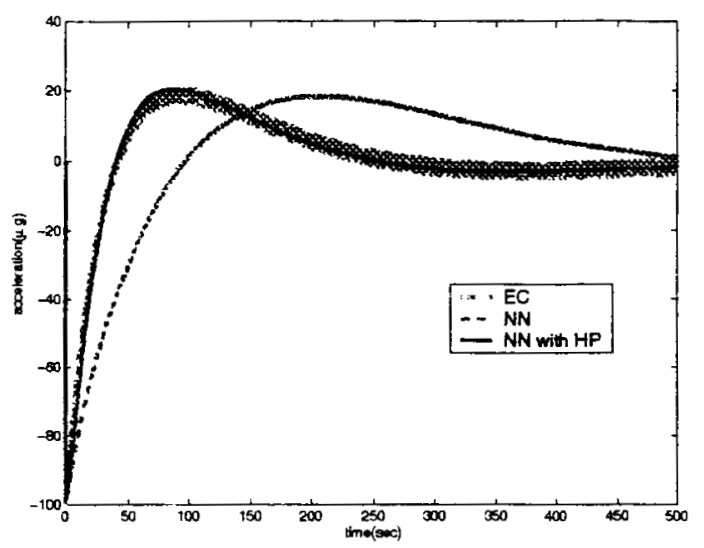

(a) Time Response of the Acceleration $y_{1}$

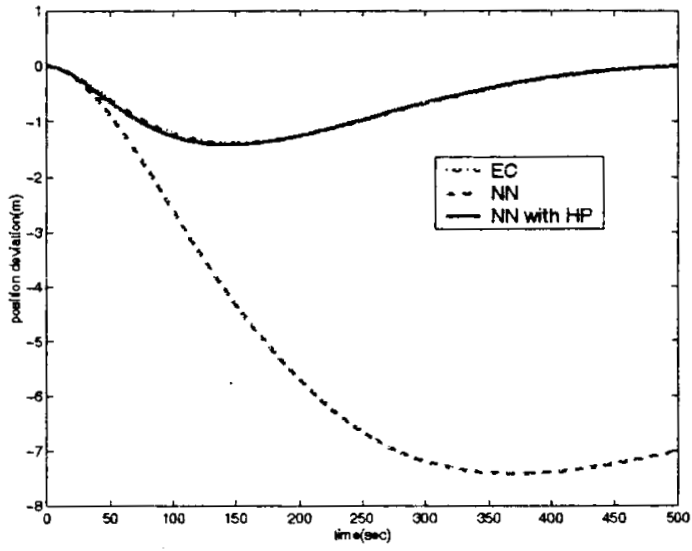

(b) Rime Response of the Position $y_{2}$

Figure 12. Acceleration and Position Responses with the $1 \mathrm{~Hz}$ base excitation

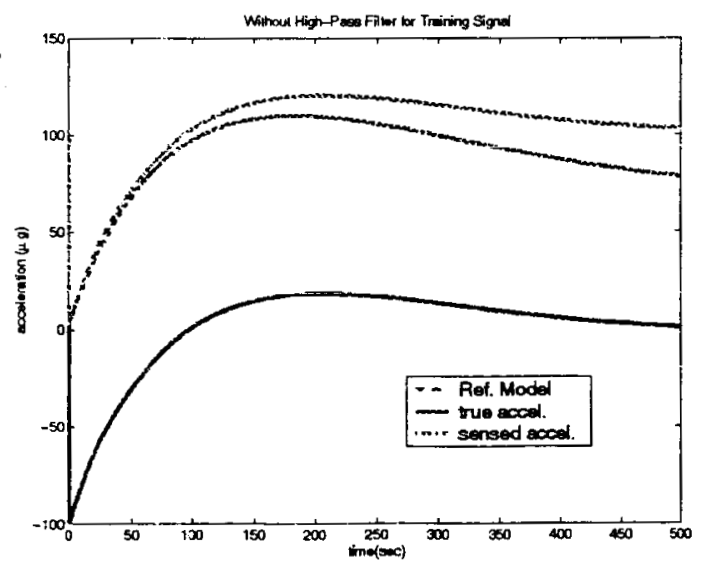

(a) The Acceleration Responses without the HighPass Filter

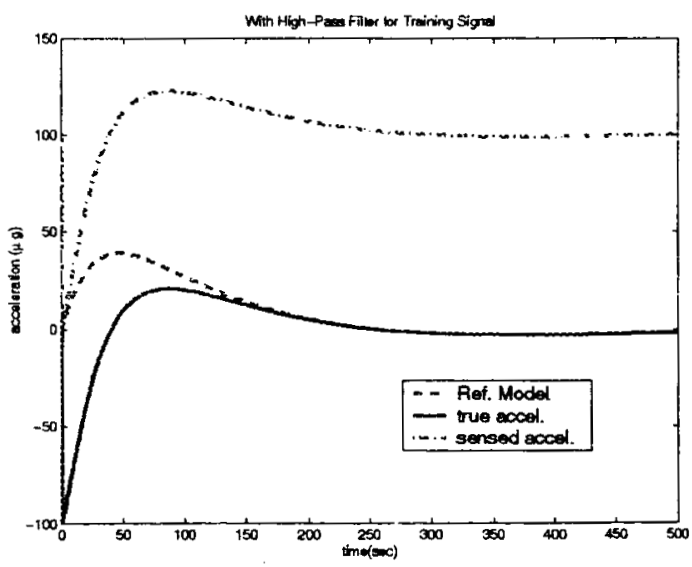

(b) The Acceleration Responses with the High-Pass Filter

Figure 13. Responses of Inner-Loop Reference Model, the Acceleration, and the Sensed Acceleration 
of a performance measure that quantify how much the transmitted acceleration is attenuated in nonlinear system and is a topic of on-going research. Simulation studies with various frequencies of base excitations show that the adaptive control outperforms the existing control system at least between $0.1 \mathrm{~Hz}$ and $5 \mathrm{~Hz}$ and achieves the same level of performance in other frequencies. Considering that active isolation is only required in the range of $0.01 \mathrm{~Hz}-10 \mathrm{~Hz}$ (The base excitations below $0.01 \mathrm{~Hz}$ must be transmitted to the isolated platform in order for the isolated system to move with the vehicle, and the open-loop system satisfies the performance requirement after $10 \mathrm{~Hz}$ ), this implies that the adaptive control system promises main benefits in the critical frequency ranges, in which parametric uncertainties related to the inertia properties and umibilical stiffness are expected to change the shape of frequency responses shown in Figure 4.

Figure 14 shows the acceleration responses when the direct force $d_{1}$ is applied as $d_{1}=160 \mu g \sin (2 \pi 0.1(H z) t)$ and $d_{1}=160 \mu g \sin (2 \pi 0.1(H z) t)$ respectively. The direct disturbance $d_{1}$ could result when the experiment
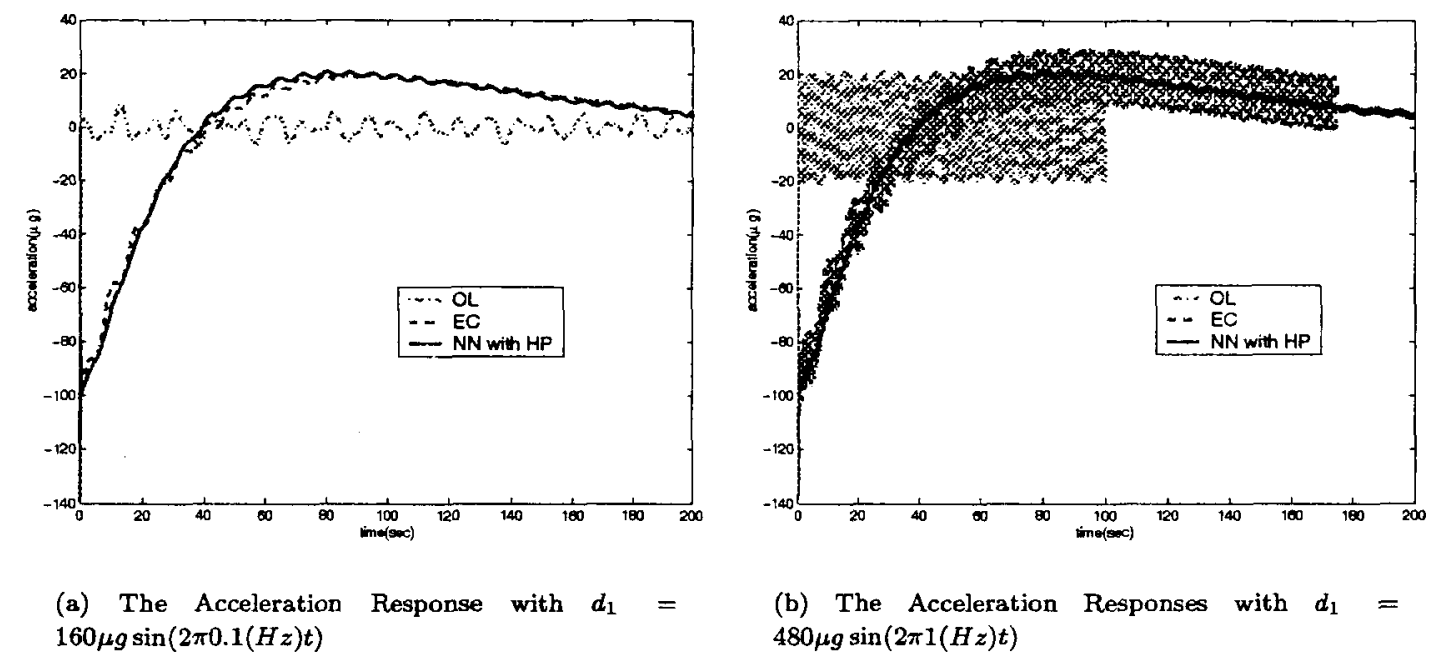

Figure 14. Responses of Inner-Loop Reference Model, the Acceleration, and the Sensed Acceleration

mounted on the isolated platform has its own source of vibration. With the adaptive control, acceleration is greatly suppressed around $1 \mathrm{~Hz}$, which is similar to the performance improvement, in the case of $1 \mathrm{~Hz}$ base excitation, shown in Figure 12(a). Similarly as the case for the base excitation, the adaptive control generally improves the performance of the existing control system between $0.1 \mathrm{~Hz}$ and $5 \mathrm{~Hz}$.

The additional disturbance $d_{2}$ might be present when the experiment is subject to the external disturbance source. In simulation, the effect of $d_{2}$ to the acceleration $y_{1}$ is in general small and has insignificant influence on the isolation system when the magnitude of $d_{2}$ is the same level as the base excitation $\ddot{x}_{o}$.

\section{Conclusions and Future Work}

We consider adaptive control augmentation of an existing linear controller for g-LIMIT. The existing control system consists of a high-gain PI acceleration controller and a low-gain PID position controller, and the adaptive control is augmented for the inner-loop acceleration controller. Introducing a high-pass filter for the NN teaching signal allows for the NN only to adapt to high-frequency dynamics and thus mitigates the effect of the accelerometer bias in adaption. This, in turn, allows for the adaptive control system maintain the deviation of the platform in the sway space at the same level of the existing control system. The adaptive control system outperforms the existing control system in the range of $0.1 \mathrm{~Hz}$ and $5 \mathrm{~Hz}$ and achieves the 
same level of performance in other frequencies.

we plan to extend the current SISO result to a MMMO system in a more realistic simulation environment for g-IMIIT provided by NASA Marshall Flight Center. On tinal submission, the paper will present the methodology and simulation results for a 6 degree-of-freedom g-LIMIT model.

\section{Acknowledgments}

This research is supported by the NASA Marshall Space Flight Center, under grant number. The authors would like to thank Dr. Young Kim in MSFC and Nathan Graybeal at Georgia Tech. for their collaboration on this research.

\section{References} 1993.

${ }^{1}$ Del Basso, S. and Bogert, P. B., "The Space Station Freedom Microgravity Environment," AIAA-1993-0831, January

${ }^{2}$ Bushnell, G. S., Anderson, T. M., Becraft, M. D., and Jacot, A. D., "Microgravity Performance Flight Characterization of an International Space Station Active Rack Isolation Prototype System," AIAA/ASME/ASCE/AHS/ASC Structures, Structural Dynamics, and Materials Conference and Exhibit, 38th, and AIAA/ASME/AHS Adaptive Structures Forum, AIAA 1997-1203, Kissimmee, FL, April 1997.

${ }^{3}$ Bushnell, G. S. and Becraft, M. D., "Microgravity Performance Flight Characterization of an International Space Station Active Rack Isolation Prototype Systern," Proceedings of the 16th IEEE Instrumentation and Measurement Technology Conference, Vol. 1, May 1999, pp. $260-267$.

${ }^{4}$ Moss, L., Just, M., Grodsinsky, C., Heese, J., and Humphreys, B., "Microgravity Environment Predictions and Control for the Fluids Integrated Rack," AIAA Aerospace Sciences Meeting, AIAA 2004-5019, Reno, NV, January 2004.

${ }^{5} \mathrm{Kim}, \mathrm{Y}$. and Whorton, M. S., "Equations of Motion for the g-LIMIT Mierogravity Vibration Isolation System," Tech. Rep. NASA TM-1999-209009, January 1999.

${ }^{6}$ Grodsinsky, C. M. and Whorton, M., "A Survey of Active Vibration Isolation Systems for Microgravity Applications," AIAA Journal of Spacecraft and Rockets, Vol. 37, No. 8, Sep.-Oct. 2000.

${ }^{7}$ Calhoun, P. C. and Hampton, R. D., "Optimal Control Design using an $\mathrm{H}_{2}$ Method for the Glovebox Integrated Microgravity Isolation Technology (g-LIMIT)," Proceedings of AIAA guidance,navigation and control conference, AIAA 2002-5020, Monterey, CA, August 2002.

${ }^{8}$ Jackson, M., Kim, Y., and Whorton, M., "Design and Analysis of the g-LIMIT Baseline Vibration Isolation Control System," Proceedings of AIA guidance, navigation and control conference, AIAA 2002-5019, Denver, Co, August 2002.

${ }^{9}$ Whorton, M., "Robust Control for the g-LIMIT Microgravity Vibration Isolation System," AIAA Journal of Spacecraft and Rockets, Vol. 42, No. 1, 2005.

${ }^{10}$ Shkolnikov, I., Shtêssel, Y., Whorton, M. S., and Jackson, M., “Microgravity Isolation Control System Design via HighOrder Sliding Mode Control," Proceedings of the American Control Conference, Chicago, $\mathrm{IL}$, June 2000, pp. 2072-2076.

${ }^{11}$ Hovakimyan, N., Yang, B.-J., and Calise, A., "An Adaptive Output Feedback Control Methodology for Non-Minimum Phase Systems," Proceedings of Conference on Decision and Control, Las Vegas, NV, 2002, pp. 949-954.

${ }^{12}$ Yang; B.-J., Hovakimyan, N., Calise, A., and Craig, J., "Experimental Validation of an Augmenting Approach to Adaptive Control of Uncertain Nonlinear Systems," Proceedings of AlAA guidance, navigation and control conference, AIAA-2003-5715, Austin, TX, 2003.

${ }^{13}$ Hornik, N., Stinchcombe, M., and White, H., "Multilayer feedforward networks are universal approximators," Neural Networks, Vol. 2, 1989, pp. 359-366.

${ }^{14} \mathrm{Ge}$, S., Lee, T., and Harris, C., Adaptive Neural Network Control of Robotic Manipulators, World Scientific, 1998.

${ }^{15}$ Lewis, F., Jagannathan, S., and Yeşildirek, A., Neural Network Control of Robot Manipulators and Nonlinear Systems, Taylor \& Francis, 1999.

${ }^{16}$ Spooner, J. T., Maggiore, M., Ordóñez, R., and Passino, K. M., Stable Adaptive Control and Estimation for Nonlinear Systems- Neural and Fuzzy Approximator Techniques, John Wiley \& Sons, New York, NY, 2002.

${ }^{17}$ Hovakimyan, N., Nardi, F., Calise, A., and Lee, H., "Adaptive Output Feedback Control of a Class of Nonlinear Systems Using Neural Networks," International Journal of Control, Vol. 74, No. 12, 2001, pp. 1161-1169.

${ }^{18}$ Lavretsky, E., Hovakimyan, N., and Calise, A., "Upper Bounds for Approximation of Continuous-Time Dynamics Using Delayed Outputs and Feedforward Neural Networks," IEEE Transactions on Automatic Control, Vol. 48, No. 9, 2003, pp. 16061610 .

${ }^{19} \mathrm{Kim}$, N., Calise, A. J., Hovakimyan, N., Prasad, J., and Corban, J. E., "Adaptive Output Feedback for High-Band width Flight Control," AIAA Journal of Guidance, Control \& Dynamics, Vol. 25, No. 6, 2002, pp. 993-1002. 
${ }^{20}$ Calise, A., Yang, B.-J., and Craig, J., "An Augmenting Adaptive Approach to Control of Flexible Systems," AIAA Journal of Guidance, Control \& Dynamics, Vol. 27, No. 3, 2004, pp. 387-396.

${ }^{21}$ Yang, B.-J., Calise, A., and Craig, J., "Adaptive Output Feedback Control of a Flexibie Base Manipulator," Proceedings of AlAA guidance, navigation and control conference, AIAA-2004-5322, Providence, RI, August 2004.

${ }^{22}$ Calise, A., Hovakimyan, N., and Idan, M., "Adaptive Output Feedback Control of Nonlinear Systems using Neural Networks," Automatica, Vol. 37, No. 8, 2001, pp. 1201-1211.

${ }^{23}$ Hovakimyan, N., Nardi, F., Kim, N., and Calise, A., "Adaptive Output Feedback Control af Uncertain Systems using Single Hidden Layer Neural Networks," IEEE Transactions on Neural Networks, Vol. 13, No. 6, 2002.

${ }^{24}$ Isidori, A., Nonlinear Control Systems, Springer-Verlag, Berlin;New York, 3rd ed., 1995. 